\title{
Biological diagnosis and monitoring of neurosyphilis: study of cerebrospinal fluid in 30 cases
}

\begin{abstract}
Aim: identify the most informative cerebrospinal fluid (CSF) markers in the diagnosis of active neurosyphilis and in surveillance during antibiotic treatment.

Type of study: retrospective, analytical, epidemiological study over a period of 10years, from 1 January 2006 to 31 December 2016 conducted in the laboratory of microbiology of CHU HASSAN II of Fez.

Patients and methods: We have identified 30 cases of neurosyphilis over a 10-year period. All patients were HIV negative. We realised on the CSF, a cytochemical examination, white and red blood cell count, a total protein and glucose levels on CSF and serum, VDRL and TPHA tests for the detection of specific antibodies.

Results: The CSF study showed 6 cases $(20 \%)$ of meningitis with cytology varying between 20 and 890 cells $/ \mathrm{ml}$ predominantly lymphocytic. $40 \%$ of cases had hyperproteinuria with extremes 0.2 and $4.3 \mathrm{~g} / 1$, glycorachia was normal in all cases. The initial titration of VDRL and TPHA was positive in $18(60 \%)$ and $22(74 \%)$ in the CSF, respectively, and $20(67 \%)$ and $26(87 \%)$ in the serum.

Conclusion: An accurate and simple approach to the diagnosis of neurosyphilis remains elusive and the diagnosis continues to require a thorough assessment of the patient, including exposure to the risk, presence of compatible clinical signs and symptoms, and laboratory. While direct tests (such as microscopy or PCR) are useful in early infection, serological testing remains the mainstay of the diagnosis of neurosyphilis.
\end{abstract}

Keywords: neurosyphilis, cerebrospinal fluid, treponema antibodies, protein

\author{
Volume 7 Issue 4 - 2017
}

Taki Imrani Zineb,' Mahha Mariam,' Hendi Rajae,' Yahyaoui Ghita,' Mahmoud Mustapha,' Benbrahim Fouzia, ${ }^{2}$ Belahsen Mohammed Faouzi

'Department of microbiology, universitary hospital Hassan II, Morocco

${ }^{2}$ Department of neurology, universitary hospital Hassan II, Morocco

Correspondence: Taki Imrani Zineb, Department of microbiology, universitary hospital Hassan II, 30000 Fes, Morocco, Email zinebtakil988@gmail.com

Received: July 30, 2017 | Published: October 30, 2017

\section{Introduction}

Syphilis is a sexually transmitted infection currently on the rise. The causative agent is a spirochete, Treponema pallidum, which in the absence of treatment can invade the central nervous system and its envelopes by creating a strong chronic inflammatory reaction: it is neurosyphilis that present the most severe neurological complication of syphilis active.

\section{Materials and methods}

A retrospective study of 30patients with Neurosyphilis over a period of 10years (from January 2006 to December 2016). The criteria for the inclusion of neurological manifestations, evocative imaging, the positivity of syphilitic serology (VDRL and TPHA) in cerebrospinal fluid (CSF) and blood. The data was collected by an exploitation sheet.

\section{Results}

The average age of our patients is 48years. All our patients are male. The average consultation period is 1.5 years. The history of syphilitic chancre was found in 10 of the young. The mean time to onset of Neurosyphilis after the chancre was 12 years. Most patients $(46 \%)$ are referred by a psychiatrist, $20 \%$ are referred by their families, and $20 \%$ are referred by an ophthalmologist, while $12.5 \%$ are referred by a primary care physician.

13 cases $(43 \%)$ were consulted for epilepticus status, 7 cases (23\%) presented for dementia, 6patients (20\%) had bilateral blindness and 3 other neurological symptoms, and one case of cerebrovascular syphilis (Table 1).

Human Immunodeficiency Virus (HIV) serology is negative for all of our patients. We realized on the CSF, a cytochemical examination with white and red blood cell count, protein and glucose assay on CSF and serum, VDRL and TPHA tests for the detection of specific antibodies.

The CSF study showed 6 cases (20\%) of meningitis with a variant of cytology between 20 and 890 elements $/ \mathrm{ml}$ predominantly lymphocyte. $40 \%$ of cases have hyperproteinorrachia with extremes of 0.6 and $4.3 \mathrm{~g} / 1$, glycorrhachia was normal in all cases. The initial titration of VDRL and TPHA was respectively positive in 18 cases $(60 \%)$ and 22 cases $(74 \%)$ in the CSF, and in 20 cases $(67 \%)$ and 26 cases $(87 \%)$ in the serum (Table 2). All patients were treated with intravenous penicillin G. Eighteen patients were cured with a two-year follow-up. Six patients are still being referred to the neurology department. Three patients were lost to follow-up. Despite the treatment, the evolution was towards a general paralysis with the deaths of three patients. 
Table I Summarizing the data of our patients at admission

\begin{tabular}{|c|c|c|c|}
\hline Patients & Age & $\begin{array}{l}\text { Antecedents } \\
\text { of syphilitic } \\
\text { chancres }\end{array}$ & Clinical signs \\
\hline I & 60 & - & Dementia \\
\hline 2 & 54 & + & Dementia \\
\hline 3 & 61 & - & Epilepticus status \\
\hline 4 & 52 & + & Bilateral blindness \\
\hline 5 & 65 & - & Dementia \\
\hline 6 & 45 & Unknown & Epilepticus status \\
\hline 7 & 64 & Unknown & Bilateral blindness \\
\hline 8 & 55 & + & Cerebrovascular disease \\
\hline 9 & 41 & - & Tabes dorsalis \\
\hline 10 & 40 & Unknown & Dementia \\
\hline II & 48 & Unknown & Epilepticus status \\
\hline 12 & 26 & - & Epilepticus status \\
\hline 13 & 32 & - & Bilateral blindness \\
\hline 14 & 21 & - & Epilepticus status \\
\hline 15 & 19 & & Epilepticus status \\
\hline 16 & 55 & + & Epilepticus status \\
\hline 17 & 64 & + & Bilateral blindness \\
\hline 18 & 30 & - & Epilepticus status \\
\hline 19 & 66 & - & Tabes dorsalis \\
\hline 20 & 53 & + & Epilepticus status \\
\hline 21 & 50 & + & Dementia \\
\hline 22 & 33 & - & Dementia \\
\hline 23 & 61 & - & Epilepticus status \\
\hline 24 & 56 & + & Epilepticus status \\
\hline 25 & 51 & + & Bilateral blindness \\
\hline 26 & 50 & - & Dementia \\
\hline 27 & 49 & + & Tabes dorsalis \\
\hline 28 & 51 & - & Epilepticus status \\
\hline 29 & 52 & - & Epilepticus status \\
\hline 30 & 36 & + & Bilateral blindness \\
\hline
\end{tabular}

Table 2 Cerebrospinal fluid profile in the diagnosis of neurosyphilis

\begin{tabular}{|c|c|c|c|c|}
\hline Patients & $\begin{array}{l}\text { White cells/ } \\
\mathrm{mm}^{3}\end{array}$ & $\begin{array}{l}\text { Red cells/ } \\
\mathbf{m m}^{3}\end{array}$ & Proteinorrachia & VDRL \\
\hline 1 & 20 & 35 & 2 & $\mathrm{I} / 4$ \\
\hline 2 & 7 & 250 & 0,77 & $1 / 4$ \\
\hline 3 & $<03$ & 1200 & 0,63 & $1 / 8$ \\
\hline 4 & 20 & 700 & 0,2 & $1 / 8$ \\
\hline 5 & 8 & 130 & 0,2 & $1 / 16$ \\
\hline 6 & 47 & 349 & 0,3 & $1 / 32$ \\
\hline 7 & 15 & 150 & 0,2 & $1 / 16$ \\
\hline 8 & 18 & 49 & 0,3 & $1 / 32$ \\
\hline 9 & 9 & 25 & 1,22 & $1 / 8$ \\
\hline 10 & 35 & 120 & $\mathrm{I}, 25$ & $1 / 64$ \\
\hline 11 & 10 & 70 & 0,68 & $1 / 64$ \\
\hline 12 & $<3$ & 80 & I, 3 & $1 / 4$ \\
\hline 13 & 6 & 34 & 4,3 & $1 / 16$ \\
\hline 14 & $<3$ & 120 & 1,5 & $1 / 32$ \\
\hline 15 & $<3$ & 65 & 0,80 & $1 / 8$ \\
\hline 16 & 17 & 78 & I & $1 / 4$ \\
\hline 17 & 890 & 220 & 4,1 & $1 / 64$ \\
\hline 18 & 20 & 53 & 0,2 & $\mathrm{I} / 4$ \\
\hline 19 & 240 & 1333 & 3,5 & $1 / 64$ \\
\hline 20 & 5 & 11 & - & $\mathrm{I} / 4$ \\
\hline 21 & 13 & 45 & - & $1 / 8$ \\
\hline 22 & 7 & 32 & - & $1 / 4$ \\
\hline 23 & 12 & 50 & 0,2 & $1 / 4$ \\
\hline 24 & 14 & 24 & 0,1 & $\mathrm{I} / 4$ \\
\hline 25 & $<3$ & 63 & 0,3 & $\mathrm{I} / 4$ \\
\hline 26 & 10 & 54 & 0,2 & $\mathrm{I} / 4$ \\
\hline 27 & 18 & 118 & 0,2 & $1 / 32$ \\
\hline 28 & $<3$ & 43 & 0,3 & $1 / 4$ \\
\hline 29 & $<3$ & 23 & 0,1 & $1 / 8$ \\
\hline 30 & $<3$ & 65 & 0,2 & $1 / 8$ \\
\hline
\end{tabular}

\section{Discussion}

Syphilis is a worldwide venereal transmission disease. It evolves in 3 phases. The primary phase is characterized by a single chancre which heals spontaneously in 4 to 6weeks. The secondary phase corresponds to the dissemination phase of the treponemes. It begins about 2 months after the contagion and presents a diffuse rash, which frequently involves the palms of the hands and soles of the feet and which disappears spontaneously in 1 to 2years. In latent syphilis, which can last for years, there are few or no symptoms. In tertiary syphilis there are gummas (soft non-cancerous growths), visceral, neurological, or heart symptoms. ${ }^{1,2}$

The diagnosis of active neurosyphilis is difficult especially in the context of old syphilis. The diagnosis of active neurosyphilis focuses on both non-specific and specific biological abnormalities of CSF. $^{3}$ The cytochemical, cytological, immunological and serological 
analyzes of CSF are useful for diagnosis. The calculation of the indexes helps to evaluate the intrathecal syntheses of the $\operatorname{IgG}, \operatorname{IgA}$ and $\operatorname{IgM}$ immunoglobulins which sign an active infection of the central nervous system. In the diagnosis of active neurosyphilis, the cell reaction and the proteinurrachia are non-specific inflammatory markers but of great sensitivity. They are indicative of an active infection before any antibiotic treatment. ${ }^{4}$ The cell reaction is always moderate to 50 elements on average. Hyperproteinorrachia is on average $1 \mathrm{~g} / \mathrm{l}$. In our series, $20 \%$ of the patients had meningitis with a predominance of lymphocytes in the CSF, and $40 \%$ had hyperproteinorrachia. The increase in IgG, IgM and IgA indexes and the presence of oligoclonal IgG bands are the most sensitive markers of central nervous system infection. Some authors consider the diagnosis of neurosyphilis in patients with positive syphilitic serology with one or more of the following CSF abnormalities: pleocytosis, hyperproteinorrachia, high level of IgG, or the presence of oligoclonal bands. In our series, these immunological tests were not used. The serology of syphilis is performed in the CSF and serum, in order to compare the titers and to calculate the specific antibody indexes. It includes a non-specific and a specific test. ${ }^{3}$ A confirmation is made by titration in case of positivity.

VDRL (Venereal Disease Research Laboratory) is a non-specific agglutination test using cardiolipin, lecithin and cholesterol as an antigen. It stills positive in the absence of treatment and becomes negative after an effective treatment. The positive reaction is followed by titration by successive dilutions of serum and CSF.

TPHA (Treponema Pallidum Haemagglutination Assay) uses a T. pallidum lysate. This is a simple and inexpensive test. The positivity threshold in the serum is fixed at 80 . The positive reaction is followed by a titration by successive dilutions of the serum and the CSF. TPHA becomes positive 35 to 45 days after infection and rarely normalizes even after a correct treatment. The title does not reflect the activity of the disease and does not help to judge its evolution. TPHA performed on the CSF has a strong negative predictive value. A positive test does not confirm the diagnosis of active neurosyphilis. To compensate for this, the Klapper specific antibody index is calculated. It is the best reflection of a local infection. ${ }^{5}$

Klapper index or TPHA index=TPHA title ratio $(\mathrm{CSF} / \mathrm{blood}) /$ albumin ratio (CSF/blood). If the index is greater than 2, an intrathecal synthesis of anti-treponemal antibodies is asserted.

FTA abs (fluorescent treponema antibody absorbed) is a specific test of indirect immunofluorescence using slides covered with a suspension of Treponema pallidum and an anti-globulin serum. Prior absorption of the patient is performed to remove non-specific antibodies. FTA-abs is positive 25 to 30 days after contamination. This is the earliest test.

There is no Gold standard for the diagnosis of neurosyphilis, but definitive diagnosis usually requires serological confirmation of syphilis (any stage) as well as a VDRL test of the cerebrospinal fluid. ${ }^{2}$ Most experts and guidelines no longer recommend routine lumbar puncture in all syphilis patients, but all agree that it should be indicated in patients with neurological symptoms or signs suggestive of neurosyphilis, including ophthalmic symptoms. ${ }^{6,2,8}$ However, it should be noted that the results of a CSF examination are often normal in patients with hearing impairment. ${ }^{9}$ Whenever the eye or the ear or both are involved, the patient's case is managed as a neurosyphilis regardless of CSF results. ${ }^{7}$ The available tests for the detection of syphilis in the CSF can be divided into direct detection and antibody analysis methods. PCR (Polymerase Chain Reaction) methods for the molecular detection of treponemes in CSF have been published by Hay et al. ${ }^{10}$ Subsequent studies have shown that this assay is not as sensitive as the rabbit infectivity assay, which can detect 1 to 2 viable treponemes whereas at least 10 treponemes are required for PCR positivity. ${ }^{11}$ At this stage, PCR is not a sufficiently sensitive tool for the systematic detection of T. pallidum in serum or CSF.

Antibody assays include VDRL and TPHA. The CSF VDRL test remains the norm for the diagnosis of neurosyphilis today, although it has a very high specificity $(99.8 \%)$, its sensitivity is only $50 \%$ (range $30 \%$ to $70 \%$ ). ${ }^{12}$ Therefore, a negative result of the CSF VDRL test does not exclude the presence of neurosyphilis, while a positive result strongly suggests the disease. Rare false positive VDRL results occur, and additional tests, such as FTA-ABS and syphilitic serology may represent another verification test. Patients who have a negative syphilis serology but who have a positive CSF VDRL test are unlikely to have neurosyphilis. An additional advantage of this assay is that it is quantitative, so the titration can be used to monitor the effectiveness of the treatment. The RPR test is easier to perform in CSF samples, but a recent study found that it also had a high false negative rate and there was currently insufficient data to replace the VDRL RPR test in CSF assays. ${ }^{13}$

Previous data have suggested that CSF-FTA-ABS is highly sensitive but non-specific and that a negative result may help exclude neurosyphilis. ${ }^{14}$ However, a recent systematic review revealed that the negative predictive value depended on the specificity of the test and the prevalence of neurosyphilis: the higher the prevalence, the lower the negative predictive value. ${ }^{15}$ The authors concluded that a negative result of treponemal-specific antibodies at the CSF level does not exclude the diagnosis of neurosyphilis when its clinical suspicion is high. ${ }^{15}$

Monitoring patients with neurosyphilis usually involves clinical monitoring as well as regular monitoring of serum RPR levels. ${ }^{6}$ In addition, patients with CSF abnormalities should be monitored every 6months until CSF parameters are normalized. ${ }^{6}$ In adults, CSF pleocytosis is generally the first improvement and a decline is expected to occur over approximately 6 months. The title of the VDRL should decrease (4 times in one year) if it is initially high, but it can takeyears to become negative. ${ }^{16}$ A persistent and low titre of VDRL in the CSF after treatment may warrant reprocessing, but if CSF pleocytosis and high protein levels have resolved and serum VDRL levels have not increased, it is unlikely That additional treatment is beneficial. ${ }^{16}$ Proteinorachia begins to decline in the first six months, but may take up to twoyears to return to normal. ${ }^{17} \mathrm{CSF}$ protein levels may decrease more slowly in neurologically abnormal patients than in those who are neurologically normal. ${ }^{18}$ Normalization of serum RPR predicts normalization of CSF and clinical abnormalities after neurosyphilis treatment. ${ }^{19}$ All CSF parameters normalize more slowly in HIVinfected patients. ${ }^{18}$ The possibility of therapeutic failure should be considered in case of clinical progression or increased RPR/VDRL levels by $\geq 2$ dilutions at any time or if CSF pleocytosis fails to resolve 2 years after therapy.

\section{Conclusion}

A precise and simple approach to the diagnosis of neurosyphilis remains elusive and the diagnosis continues to require a thorough assessment of the patient, including exposure to the risk, presence of symptoms and compatible clinical signs and laboratory tests. 
While direct tests (such as microscopy or PCR) are useful in early infection, serological testing remains the mainstay of the diagnosis of neurosyphilis.

\section{Acknowledgements}

None.

\section{Conflict of interest}

The author declares no conflict of interest.

\section{References}

1. Lukehart SA, Hook EW III, Baker-Zander SA, et al. Invasion of the central nervous system by Treponema pallidum: implications for diagnosis and treatment. Ann Intern Med. 1988;109(11):855-862.

2. Marra CM. Update on neurosyphilis. Curr Infect Dis Rep. 2009;11(2):127-134.

3. C Caudie, Garel F, J Bancel C, et al. Diagnostic et surveillance biologique de six neurosyphilis: apport de l'étude du liquide céphalorachidien. Ann Biol Clin. 2003;61:563-569.

4. Jordan KG, Simon RP, Miller JN. Modern neurosyphilis: immunobiology, pathogenis and clinical features. In: Roland LP editor. Merritt's textbook of neurology. update 4. Philadelphie; 1990. p. 3-20.

5. Klapper PE, Laing I, Longson M. Rapid non-invasive diagnosis of herpes encephalitis. Lancet. 1981;2(8247):607-609.

6. Centers for Disease Control and Prevention (CDC). Sexually transmitted diseases guidelines, 2010. MMWR Morb Mortal Wkly Rep. 2010;59:1-116.

7. O'donnell JA, Emery CL. Neurosyphilis: a current review. Curr Infect Dis Rep. 2005;7(4):277-284.

8. Ghanem KG. Review: neurosyphilis: a historical perspective and review. CNS Neurosci Ther. 2010;16(5):e157-e168.
9. Becker GD. Late syphilis hearing loss: a diagnostic and therapeutic dilemma. Laryngoscope. 1979;89(8):1273-1288.

10. Hay PE, Clarke JR, Strugnell RA, et al. Use of the polymerase chain reaction to detect DNA sequences specific to pathogenic treponemes in cerebrospinal fluid. FEMS Microbiol Lett. 1990;56(3):233-238.

11. Burstain JM, Grimpel E, Lukehart SA, et al. Sensitive detection of Treponema pallidum by using the polymerase chain reaction. J Clin $\mathrm{Mi}$ crobiol. 1991;29(1):62-69.

12. Larsen S, Johnson RE. Diagnostic tests. 1998. p. 1-27.

13. Marra CM, Tantalo LC, Maxwell CL, et al. The rapid plasma reagin test cannot replace the venereal disease research laboratory test for neurosyphilis diagnosis. Sex Transm Dis. 2012;39(6):453-457.

14. Jaffe HW, Larsen SA, Peters M, et al. Tests for treponemal antibody in CSF. Arch Intern Med. 1978;138(2):252-255.

15. Harding AS, Ghanem KG. The performance of cerebrospinal fluid treponemal-specific antibody tests in neurosyphilis:a systematic review. Sex Transm Dis. 2012;39(4):291-297.

16. Jordan KG. Modern neurosyphilis- a critical analysis. West $J$ Med. $1988 ; 149(1): 47-57$

17. Flores JL. Syphilis. A tale of twisted treponemes. West $J$ Med. 1995;163(6):552-559.

18. Marra CM, Longstreith WT, Maxwell CL, et al. Resolution of serum and cerebrospinal fluid abnormalities after treatment of neurosyphilis. Influence of concomitant human immunodeficiency virus infection. Sex Transm Dis. 1996;23(3):184-189.

19. Marra CM, Maxwell CL, Tantalo LC, et al. Normalization of serum rapid plasma reagin titer predicts normalization of cerebrospinal fluid and clinical abnormalities after treatment of neurosyphilis. Clin Infect Dis. 2008;47(7):893-899. 